UDC 515:142

\title{
On Homology Groups of a Subspace of Triangulations of the Two-Simplex with not More than 6 Subdivisional Boundary Vertices
}

\author{
Yablokova S.I. \\ P.G. Demidov Yaroslavl State University, \\ Sovetskaya str., 14, Yaroslavl, 150000, Russia \\ e-mail: yabl@uniyar.ac.ru \\ received October 20, 2013
}

Keywords: triangulation, simplex, homology group

We study homology groups of the space $\tilde{W}_{1}\left(\nabla^{N}\right)$ of triangulations of the twodimensional simplex with vertices $D_{0} D_{1} D_{2}$ endowed with a boundary subdivision with not more than 6 vertices in case when this boundary subdivision is extended to the interior of the simplex without adding new interior vertices. As a result, we obtain a theorem about the homology groups $H_{n}$ in cases $n=0, \ldots, 5$.

The article is published in the author's wording.

\section{Introduction}

Let us consider the two-dimensional simplex $\sigma^{2}$ with vertices $D_{0} D_{1} D_{2}$ endowed with a boundary subdivision that contains three old vertices of the simplex. Assume that this subdivision contains at most $N$ new vertices and, moreover, that the edge $D_{0} D_{1}$ contains at most $p$ new vertices, $D_{1} D_{2}$ contains at most $q$ new vertices, $D_{2} D_{0}$ contains at most $s$ new vertices, where $p, q, s$ are non-negative integers, $p+q+s=n, 0 \leq n \leq N$. Such a subdivision will be said to be of type $(p, q, s)$. Here we are considering the triangulations of the simplex of the special type. The boundary subdivision is extended to the interior of the simplex without adding new interior vertices and the obtained triangulations don't contain the interior subsimplex with the vertices lying on all three edges of $\sigma^{2}$.

Our goal is to find the homology groups of the space of these triangulations.

\section{Construction of the space of triangulations}

Let us identify the set of the boundary subdivision of the simplex $\sigma^{2}$ with a polyhedron $\nabla_{p, q, s}^{n}$. As a result we have the natural surjective mapping

$$
\pi: \operatorname{Tr}(p, q, s) \rightarrow \nabla_{p, q, s}^{n}
$$


assigning to any triangulations the corresponding boundary subdivision. It is not bijective since there are many ways of extending a given boundary subdivision to the interior of the simplex. Thus we may regard $\operatorname{Tr}(p, q, s)$ as a (ramified) finite sheeted covering $\tilde{\nabla}_{p, q, s}^{n}$ over each polyhedron $\nabla_{p, q, s}^{n}$. It was proved in [1] that the number $T_{k}$ of different triangulations lying over an open $k$-cell $\stackrel{\circ}{\nabla}_{p, q, s}^{k}(p+q+s=k)$ of the polyhedron $\nabla_{p, q, s}^{n}$ in the case when we don't consider the triangulations with the interior subsimplex with the vertices on all three edges of $\sigma^{2}$ is given by the formula

$$
T_{k}=C_{k-1}^{p-1}+C_{k-1}^{q-1}+C_{k-1}^{s-1}
$$

(Note that $T_{0}=T_{1}=1$.)

The space of such triangulations of the 2-dimensional simplex $\sigma^{2}$ is a cell complex $\tilde{W}_{1}\left(\nabla^{N}\right)$.

\section{Construction of the incidence matrixies}

In order to find the homology groups let us use the chain complex $C=\oplus_{n} C_{n}$ corresponding to our $\mathrm{CW}$-space and construct the incidence matrixies ${ }^{n} E$, defyning the boundary mapping $C_{n+1} \rightarrow C_{n}$. Left index of the incidence matrix contains all $n$-dimensional cells (sheets of the covering) of the polyhedron $\tilde{\nabla}_{p, q, s}^{n}(p+q+s=n, \quad p, q, s \geq 0)$, upper index contains all $(n+1)$-dimensional cells (sheets) of the polyhedron $\tilde{\nabla}_{p, q, s}^{n+1}$ $(p+q+s=n+1, \quad p, q, s \geq 0)$, each cell has a fixed orientation. In the intesection of the line $i$ and column $j$ we put the incidence coefficient of the $(n+1)$-dimensional $j$-cell and $n$-dimensional $i$-cell. The number of the cell is defined in such a way that the incidence matrix has the most simple block structure. It has non-zero blocks on the left-right diagonal of the matrix and some non-zero blocks near this diagonal. To get this block structure we had to define the special order of the $n$-dimensional cells (sheets) in the basis of the group $C_{n}$.

The first cells in the basis of $C_{n}$ are $\nabla_{n, 0,0}^{n}, \nabla_{0, n, 0}^{n}, \nabla_{0,0, n}^{n}$, consisting the block ${ }_{n}^{n} \mathcal{A}$. The next block of cells ${ }_{n-1}^{n} \mathcal{A}$ contains sheets of the coverings $\tilde{\nabla}_{n-p, p, 0}^{n}$ and $\tilde{\nabla}_{p, n-p, 0}^{n} \quad(1 \leq$ $p \leq k, 2 k=n, 2 k+1=n)$ in order

$$
\tilde{\nabla}_{n-1,1,0}^{n}, \tilde{\nabla}_{1, n-1,0}^{n}, \tilde{\nabla}_{n-2,2,0}^{n}, \tilde{\nabla}_{2, n-2,0}^{n}, \ldots, \tilde{\nabla}_{k, k, 0}^{n}
$$

then the sheets of the coverings $\tilde{\nabla}_{n-p, 0, p}^{n}$ and $\tilde{\nabla}_{p, 0, n-p}^{n} \quad(1 \leq p \leq k)$ and at last - the sheets of the coverings $\tilde{\nabla}_{0, n-p, p}^{n}$ and $\tilde{\nabla}_{0, p, n-p}^{n} \quad(1 \leq p \leq k$.) (The order of these sheets is similar to (1)). Each covering $\tilde{\nabla}_{p, q, 0}^{n}\left(\tilde{\nabla}_{p, 0, q}^{n}, \tilde{\nabla}_{0, p, q}^{n}\right) \quad p+q=n, \quad p, q \geq 0$, contains $C_{n}^{p}$ $n$-dimensional cells (sheets) of the CW-space $\tilde{W}_{1}\left(\nabla^{N}\right)$. These cells are numbered in the following way.

In [1] it has been proved that each extension of a given boundary subdivision to the interior of the simplex $\sigma^{2}$ is defined by the weights of the subdivisional boundary vertices. By the weight of the boundary vertice we call the number of the interior divisional segments connecting this vertice with other divisional vertices of the boundary. In case when we have subdivisional vertices only on the two edges of the simplex the sum of the weights on one of the two edges of the simplex is equal to $n$. Consequently the first 
$n$-cells in the basis of $C_{n}$ are defined by the following sequences of weights:

$$
\begin{gathered}
\left(n-p+1,1, \ldots, 1 ; v_{1}^{(1)}, \ldots, v_{q}^{(1)} ; 0\right) \\
\left(1, n-p+1, \ldots, 1 ; v_{1}^{(2)}, \ldots, v_{q}^{(2)} ; 0\right) \\
\ldots \ldots \ldots+\ldots . . . . . \\
\left(1,1, \ldots, n-p+1 ; v_{1}^{(p)}, \ldots, v_{q}^{(p)} ; 0\right) .
\end{gathered}
$$

The next $n$-cells are defined by the following sequences of weights:

$$
\begin{gathered}
\left(n-p, 2, \ldots, 1 ; \tilde{v}_{1}^{(1)}, \ldots, \tilde{v}_{q}^{(1)} ; 0\right) \\
\left(n-p, 1,2, \ldots, 1 ; \tilde{v}_{1}^{(2)}, \ldots, \tilde{v}_{q}^{(2)} ; 0\right) \\
\ldots \ldots \ldots . \ldots . \ldots . . . \\
\left(n-p, 1, \ldots, 2 ; \tilde{v}_{1}^{(p-1)}, \ldots, \tilde{v}_{q}^{(p-1)} ; 0\right) \\
\left(2, n-p, 1, \ldots, 1 ; \tilde{v}_{1}^{(p)}, \ldots, \tilde{v}_{q}^{(p)} ; 0\right) \\
\left(1, n-p, 2, \ldots, 1 ; \tilde{v}_{1}^{(p+1)}, \ldots, \tilde{v}_{q}^{(p+1)} ; 0\right) \\
\ldots \ldots \ldots \ldots . \ldots . \ldots . \\
\left(1,1, \ldots, 2, n-p ; \tilde{v}_{1}^{\left(p^{2}-p\right)}, \ldots, \tilde{v}_{q}^{\left(p^{2}-p\right)} ; 0\right) .
\end{gathered}
$$

The next $n$-cells are defined by the sequences of weights where one of them is equal to $n-p-1$, one is equal to 3 and all others on this edge have a weight 1 . And so on we are numbering the cells in the same way, i.e. deducting $u_{1}$ by 1 , fixing the set $u_{2}, u_{3}, \ldots, u_{p}$ so that $u_{1}+\cdots+u_{p}=n, \quad u_{1} \geq u_{2} \geq \cdots \geq u_{p}$ and considering all various rearrangments of the set. Then the same process we apply to the set of the weights $v_{1}, \ldots, v_{q}$.

The next set of blocks of $n$-cells in the basis of $C_{n}$ are blocks of type $(p, q, s)$, $p, q, s>0$ :

$$
\tilde{\nabla}_{p, q, s}^{n}, \tilde{\nabla}_{p, s, q}^{n}, \tilde{\nabla}_{q, p, s}^{n}, \tilde{\nabla}_{s, p, q}^{n}, \tilde{\nabla}_{q, s, p}^{n}, \tilde{\nabla}_{s, q, p}^{n}, \quad p+q+s=n, \quad p \geq q \geq s
$$

These blocks of cells follow in the order of lessening of $q$. In each covering $\tilde{\nabla}_{p, q, s}^{n}(p+q+s=$ $n, p, q, s>0$ ) we are numbering $n$-cells corresponding to the triangulations of the simplex $\sigma^{2}$ with maximum sum of the weights of subdivisional vertices of one of the edges. This part contains $C_{n-1}^{p-1}+C_{n-1}^{q-1}+C_{n-1}^{s-1} n$-cells (sheets) of $\tilde{W}_{1}\left(\nabla^{N}\right)$. The cells are numbered in the same way as in the block ${ }_{n-1}^{n} \mathcal{A}$. The only difference here: we have subdivisional vertices on all the edges, so we have to consider the third set of the weights $t_{1}, \ldots, t_{s}$. This third set changes in the same way as the first two sets.

The basis of the group $C_{n+1}$ is constructed in the same way.

The main difficulty here is the dimensions of the incidence matrix. Even if $n$ is small the martix ${ }^{n} E$ is quite big. ${ }^{0} E$ is a zero matrix with one line and 3 columns. ${ }^{1} E$ is a $3 \times 9$ matrix:

$$
\left(\begin{array}{ccccccccc}
-1 & 0 & 0 & 0 & -1 & 0 & 1 & & \\
& -1 & 0 & -1 & 0 & 0 & 0 & 0 & -1 \\
& & -1 & 0 & 0 & 1 & 0 & -1 &
\end{array}\right)
$$


${ }^{2} E$ has 9 lines and 24 columns. ${ }^{3} E$ is $24 \times 60$ matrix with the following block structure:

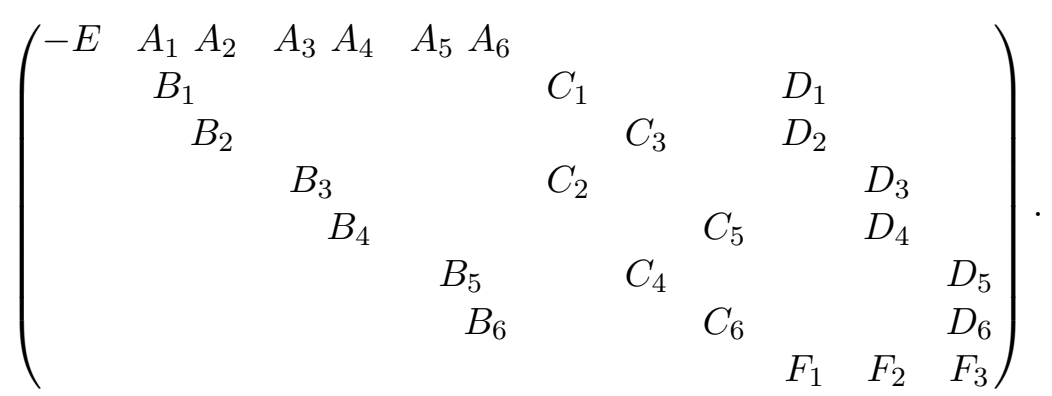

Here $-E=\operatorname{diag}(-1,-1,-1)$, the dimensions of other blocks are: $A_{i}, B_{i}(i=1, \ldots, 6)-$ $3 \times 4 ; C_{i}(i=1, \ldots, 6)-3 \times 6 ; D_{i}, F_{i}(i=1, \ldots, 6)-3 \times 5$. For $n=4$ we have ${ }^{4} E$ with 60 lines and 144 columns, for $n=5{ }^{5} E$ has 144 lines and 336 columns, ${ }^{6} E$ has 336 lines and 768 columns. The incidence matrix ${ }^{4} E$ has the following block structure:

$$
\left(\begin{array}{ccccc}
0 & J_{1} J_{2} & & & \\
0 & J_{3} J_{4} & & & \\
0 & J_{5} J_{6} & & M_{1} & \\
& G_{1} & K_{1} & M_{2} & \\
& G_{2} & K_{2} & M_{3} & \\
& G_{3} & K_{3} & M_{4} & \\
& G_{4} & K_{4} & M_{5} & \\
& G_{5} & K_{5} & M_{6} & \\
& G_{6} & K_{6} & & P_{1} \\
& & L_{1} L_{3} & & P_{2} \\
& L_{2} L_{5} & & P_{3} \\
& L_{4} L_{6} & & R_{1} R_{2} \\
& & & N_{1} & R_{3} \\
& & & N_{2} & R_{5} R_{6}
\end{array}\right)
$$

here the dimensions of the blocks are: $J_{i}(i=1, \ldots, 6)-1 \times 5 ; G_{i}(i=1, \ldots, 6)-4 \times 5$; $L_{i}(i=1, \ldots, 6)-6 \times 10 ; K_{i}(i=1, \ldots, 6)-4 \times 10 ; M_{i}(i=1, \ldots, 6)-4 \times 8 ; N_{i}(i=$ $1,2,3)-5 \times 8 ; P_{i}(i=1,2,3)-6 \times 9 ; R_{i}(i=1, \ldots, 6)-5 \times 9$.

The block structure of the matrix ${ }^{5} E$ :

$$
\left(\begin{array}{cccccccc}
-E & J & & & & & \\
& A_{1} & A_{2} & B & & & \\
& & A_{3} & & C & A_{4} & \\
& & & D & & F & \\
& & & & & G & H
\end{array}\right) .
$$

Here each block also has a block structure. $D, A_{i}(i=1, \ldots, 4)$ are diagonal matrixes with non-zero blocks on the main diagonal: $D=\operatorname{diag}\left(D_{1}, D_{2}, D_{3}\right), A_{i}=\operatorname{diag}\left(A_{i 1}, \ldots, A_{i 6}\right)$. The blocks have the following dimensions: $D_{i}(i=1,2,3)-8 \times 12, A_{1 j}-5 \times 6, A_{2 j}-5 \times 15$, 
$A_{3 j}-10 \times 15, A_{4 j}-10 \times 16(j=1, . .6) . B$ and $C$ are block matrixes

$$
\left(\begin{array}{ccc}
B_{1} & & \\
B_{2} & & \\
& B_{3} & \\
& B_{4} & \\
& & B_{5} \\
& & B_{6}
\end{array}\right), \quad\left(\begin{array}{llll}
C_{1} & & \\
& C_{3} & \\
C_{2} & & \\
& & C_{5} \\
& C_{4} & \\
& & C_{6}
\end{array}\right)
$$

where $B_{i}(i=1, \ldots, 6)-5 \times 12, C_{i}(i=1, . ., 6)-10 \times 20$ matrixies.

$J$ and $F$ have the same block structure:

$$
\left(\begin{array}{cccccc}
F_{1} & F_{2} & & & & \\
& & F_{3} & F_{4} & & \\
& & & & F_{5} & F_{6}
\end{array}\right),
$$

where $F_{i}(i=1, \ldots, 6)$ is a $8 \times 16$ matrix, $J_{i}(i=1, \ldots 6)-1 \times 6$ matrix. $G$ has the following block structure:

$$
\left(\begin{array}{ccccc}
G_{1} & & G_{3} & & \\
& G_{2} & & G_{5} & \\
& & G_{4} & & G_{6}
\end{array}\right),
$$

where each $G_{i}$ is a $9 \times 16$ matrix. $H$ is a column of three $9 \times 15$ matrixies.

\section{Homology groups of the space of triangulations}

We use the following elementary transformations of the columns of the incidence matrix ${ }^{n-1} E$ :

1) exchanging of the places of the columns $i$ and $j$;

2) multiplying the column i by -1 ;

3) adding the column $j$ to the column $i$.

The coordinated transformations of the incidence matrix ${ }^{n} E$ are:

1) exchanging of the lines $i$ and $j$;

2) multiplying the line $i$ by -1 ;

3) substracting the line $i$ from the line $j$.

As a result of the coordinated transformations of the incidence matrixies ${ }^{0} E,{ }^{1} E, \ldots$, ${ }^{5} E$ of the $\mathrm{CW}$-space $\tilde{W}_{1}\left(\nabla^{N}\right)$ we obtain the ranks of these matrixies:

$\operatorname{rang}{ }^{1} E=3, \quad \operatorname{rang}{ }^{2} E=6, \quad \operatorname{rang}{ }^{3} E=18, \quad \operatorname{rang}{ }^{4} E=42, \quad \operatorname{rang}{ }^{5} E=102$

and the zero torsion coefficients.

Now we can calculate the Betti numbers with the help of the formula

$$
p_{i}=\alpha_{i}-\operatorname{rang}^{i-1} E-\operatorname{rang}^{i} E,
$$

where $\alpha_{i}$ is the number of the $i$-dimensional cells of the CW-complex:

$$
p_{1}=0, \quad p_{2}=0, \quad p_{3}=0, \quad p_{4}=0, \quad p_{5}=0 .
$$


Theorem 1. Let $\tilde{W}_{1}\left(\nabla^{N}\right)$ be a $C W$-space of triangulations of the 2-simplex $\sigma^{2}$ with at most $N$ subdivisional vertices on its edges. Assume that the boundary subdivision is extended to the interior of $\sigma^{2}$ without adding new interior vertices and triangulations don't contain interior subsimplex with the vertices on the three edges of the simplex. Then the homology groups $H_{n}$ of this space are trivial in cases $n=1,2,3,4,5$ and in case $n=0$ the homology group is a free cyclic group.

If we consider the space of triangulations of the simplex $\sigma^{2}$ with the interior subsimplex $\tau^{2}$ with vertices on all edges of $\sigma^{2}$ then the dimensions of the incidence matrixies increase, because the number of different triangulations lying over an open $k$-cell $\stackrel{\circ}{p, q, s}^{k}$ $(p+q+s=k)$ of the polyhedron $\nabla_{p, q, s}^{n}$ is given by the formula

$$
T_{k}=C_{k-1}^{p-1}+C_{k-1}^{q-1}+C_{k-1}^{s-1}+\sum_{i=1}^{p} \sum_{j=1}^{q} C_{p-i+j-1}^{p-i} C_{q+s+i-j-1}^{s-1} .
$$

In ${ }^{n} E$ we must include $n$-cells corresponding to these triangulations. They follow after the cells corresponding to the triangulations without subsimplex $\tau^{2}$. The order of these $n$-cells depends on the position of the vertices of subsimplex $\tau^{2}$. At first we include the cells corresponding to the triangulations of $\sigma^{2}$ where two first vertices of $\tau^{2}$ are the first subdivisional vertices of the edges $D_{0} D_{1}$ and $D_{1} D_{2}$ accordingly and the third vertice is going in succession through all subdivisional vertices of the edge $D_{2} D_{0}$. The numbering of $n$-cells corresponding to the triangulations with the fixed third vertice of $\tau^{2}$ is the same as it was described earlier. Then we move the second vertice of $\tau^{2}$ to the second subdivisional vertice of the edge $D_{1} D_{2}$ and once more allow the third vertice to move through all subdivisional vertices of the edge $D_{2} D_{0}$ and so on. After using all subdivisional vertices on $D_{1} D_{2}$ as the second vertice of $\tau^{2}$ we move the first vertice of $\tau^{2}$ from the first subdivisional vertice of $D_{0} D_{1}$ to the second and begin the same process again.

In this case the dimensions of the incidence matrixies are: ${ }^{0} E-1 \times 3,{ }^{1} E-3 \times 9$, ${ }^{2} E-9 \times 16,{ }^{3} E-16 \times 66,{ }^{4} E-66 \times 168,{ }^{5} E-168 \times 416$.

As a result of the coordinated transformations of the incidence matrixies ${ }^{0} E,{ }^{1} E, \ldots$, ${ }^{5} E$ of the CW-space $W_{1}\left(\nabla^{N}\right)$ we obtain Betti numbers

$$
p_{1}=0, \quad p_{2}=0, \quad p_{3}=1, \quad p_{4}=0, \quad p_{5}=0
$$

and the zero torsion coefficients.

Theorem 2. Let $W_{1}\left(\nabla^{N}\right)$ be a $C W$-space of triangulations of the 2-simplex $\sigma^{2}$ with at most $N$ subdivisional vertices on its edges. Assume that the boundary subdivision is extended to the interior of $\sigma^{2}$ without adding new interior vertices. Then the homology groups $H_{n}$ of this space are trivial in cases $n=1,2,4,5$ and in cases $n=0,3$ the homology group is a free cyclic group.[2] 


\title{
References
}

1. Yablokova S.I. Triangulyatsii dvumernogo simpleksa, vse vershyny kotoryh lezhat na ego granitse // Voprosy teorii grupp i gomologicheskoy algebry. Yaroslavl, 1994. S. $69-88$ [in Russian: Яблокова С.И. Триангуляции двумерного симплекса, все вершины которых лежат на его границе // Вопросы теории групп и гомологической алгебры. Ярославль, 1994. C. $69-88]$.

2. Yablokova S.I. Gruppy gomology nekotoryh prostranstv triangulyasty // Matematika v Yaroslavskom Universitete. Yaroslavl, 2011. S. 207-217 [in Russian: Яблокова С.И. Группы гомологий некоторых пространств триангуляций // Математика в Ярославском университете. Ярославль, 2011. С. 207 - 217].

\section{О группах гомологий одного подпространства триангуляций двумерного симплекса с не более чем 6 точками разбиения границы}

\author{
Яблокова С.И. \\ Ярославский государственный университет им. П.Г. Демидова \\ 150000 Россия, е. Ярославль, ул. Советская, 14
}

Ключевые слова: триангуляция, симплекс, группа гомологий

Рассматривается группа гомологий пространства $\tilde{W}_{1}\left(\nabla^{N}\right)$ триангуляций двумерного симплекса $D_{0} D_{1} D_{2}$ с не более чем 6 точками разбиения границы в случае, когда триангуляции с границы продолжаются на внутренность симплекса без добавления новых точек разбиения. В результате получены группы гомологий $H_{n}$ для $n=0, \ldots, 5$.

Статья публикуется в авторской редакции.

\section{Сведения об авторе:}

Яблокова Светлана Ивановна,

Ярославский государственный университет им. П.Г. Демидова, канд. физ.-мат. наук, доцент 